\title{
Registered Reports: A process to safeguard high-quality evidence
}

\author{
Anne M. Scheel \\ Human-Technology Interaction Group, \\ Eindhoven University of Technology \\ https://orcid.org/0000-0002-6627-0746 \\ a.m.scheel@tue.nl
}

This manuscript is now published at Quality of Life

Research. Please cite as

Scheel, A. M. (2020). Registered Reports: A process to safeguard

high-quality evidence. Quality of Life Research, 29(12),

3181-3182. https://doi.org/10.1007/s11136-020-02698-w

\section{Declarations}

Funding: This work was funded by VIDI grant 452-17-013.

Conflicts of interest/Competing interests: I am conducting and publishing meta-scientific research on Registered Reports in psychology.

Availability of data and material: Not applicable

Code availability: Not applicable 
From the perspective of an individual researcher, Registered Reports may appear as little more than yet another article format - a format with an unusual workflow, perhaps, but ultimately just a slightly different route for one's research to enter the published record. From the perspective of a scientific claim, however, Registered Reports are much more than that: They establish a new standard for evidence quality. A hypothesis that is upheld in a Registered Report has survived a process that was highly potent at finding any flaws with it. First, the method used to test the hypothesis was vetted in peer review and judged capable of providing an informative test before the results were known. Second, the criteria the data had to pass to be counted as supporting the hypothesis were predefined and left minimal room for the evidence to be presented as stronger than warranted (e.g., due to capitalising on chance). And third, the results would have been published in the same place even if they had contradicted the hypothesis.

Compare this to a hypothesis supported in a regular empirical article: Typically, this means that the method only received independent criticism once the results were known to all parties, which may have coloured the reviewers' judgment; that neither the test criteria nor the hypothesis itself were publicly defined a priori and (knowingly or unknowingly) may have been chosen to fit random patterns in the data; and that contradictory results might never have been submitted for publication or survived the review process. This description is not intended to accuse the research community of habitually misrepresenting their findings and massaging their publication records. Yet the fact of the matter is that our regular publication system puts almost no barriers in the way of such dynamics.

Clearly, the hypothesis published in a Registered Report has passed a much more 'severe' test, a test that had a high probability of refuting the hypothesis if it were false $[1,2]$. Because Registered Reports have transparent, results-independent selection criteria and strategically placed barriers that prevent authors and editors from getting fooled by their own biases, the evidence for scientific claims published through this route will on average be of far higher quality than that of their counterparts in the regular literature.

Critics might object that quality standards for regular articles vary across journals, and that some outlets have always required exceptionally high standards of 
evidence for the scientific claims they publish. This may be true, but taking the perspective of the scientific claim shows where the regular publication system fails: For every supporting piece of evidence published in a high-quality journal, the number of contradicting pieces of evidence that might exist but never got published remains unknown. In other words, we cannot know how lucky the authors of a successful paper in a high-quality journal were to find their results, and how many unlucky peers of theirs never made it into the published record. By allowing publication bias to exist, we deprive ourselves of an unbiased, comprehensive evaluation of the evidence, and ultimately jeopardise the integrity of our scientific knowledge.

While Registered Reports provide high-quality evidence in theory, putting them into practice is no trivial task. To work their magic, Registered Reports require an investment from both authors and editors. Authors are faced with the often unfamiliar challenge of developing detailed specifications of their hypotheses, methods, and statistical analyses before having collected their data. This may seem like a mere reshuffling of the established workflow, but experience has shown that it can be surprisingly difficult and even lead to the realisation that one's research question is critically immature [3]. Although I personally believe that the RegisteredReports format can benefit a great variety of research endeavours, it develops its full potential for mature research questions that warrant highly specific, well-defined predictions.

However, I do not want to discourage interested authors and give the false impression that Registered Reports are reserved for a small elite of extremely wellunderstood research problems. In fact, preparing a Registered-Report submission and interacting with reviewers during the Stage-1 review can provide the perfect breeding ground to develop a new idea into a testable and test-worthy prediction. But I want to caution against trivialising this task: Lapses, oversights, and excessive vagueness in the Stage-1 protocol cannot be salvaged once the data are in. The answer provided by the result of a hypothesis test can only be as informative as the question allows.

Editors, on the other hand, are responsible for safeguarding the publication process. Any set of guidelines and rules can only be effective if they are adhered to, 
and guideline adherence is rarely automatic. As an early survey of published Registered Reports showed, even crucial ingredients such as making the in-principleaccepted protocol publicly available (i.e., registering the report) are not a matter of course [4]. But Registered Reports can only produce claims with high evidential quality if their process is respected and protected from infractions: strategically placed barriers cannot keep biases in check if authors, reviewers, or editors are able to remove them at will. To ensure that a Registered Report is worth its salt, journals and editors thus need to develop clear and specific guidelines for authors, reviewers, and their own editorial team, maximise transparency of the editorial process to allow independent verification, and establish accountability for their own adherence to the guidelines, for example by providing a robust mechanism through which authors and reviewers can raise concerns.

Like any profound change to an established routine, adopting Registered Reports comes at a cost. But for all members of the research community, this investment pays off in the most valuable currency: the chance to build a more robust published record than ever before. I wish the editorial team and the contributors to Quality of Life Research the best of luck with this exciting project.

\section{References}

1. Mayo, D. G. (2018). Statistical Inference as Severe Testing: How to Get Beyond the Statistics Wars (1st ed.). Cambridge University Press. doi: https://doi.org/10.1017/9781107286184

2. Lakens, D. (2019). The value of preregistration for psychological science: A conceptual analysis. Japanese Psychological Review, 62(3), 221-230.

3. Scheel, A. M., Tiokhin, L., Isager, P. M., \& Lakens, D. (2020). Why Hypothesis Testers Should Spend Less Time Testing Hypotheses: Perspectives on Psychological Science. https://doi.org/10.1177/1745691620966795

4. Hardwicke, T. E., \& Ioannidis, J. P. A. (2018). Mapping the universe of registered reports. Nature Human Behaviour, 2(11), 793-796. doi: https://doi.org/10.1038/s41562-018-0444-y 